\title{
Advancing the conductivity-permselectivity tradeoff of electrodialysis ion-exchange membranes with sulfonated CNT nanocomposites
}

\author{
Hanqing Fan ${ }^{a}$, Yuxuan Huang ${ }^{\mathrm{a}}$, Ngai Yin Yip ${ }^{\text {a, b, * }}$ \\ ${ }^{a}$ Department of Earth and Environmental Engineering, Columbia University, New York, 10027-6623, United States \\ ${ }^{\mathrm{b}}$ Columbia Water Center, Columbia University, New York, 10027-6623, United States
}

\section{A R T I C L E I N F O}

\section{Keywords:}

Ion-exchange membrane

Carbon nanotube nanocomposite

Conductivity-permselectivity tradeoff

Percolating network

\begin{abstract}
A B S T R A C T
Ion exchange membranes, IEMs, are widely applied in water and energy technologies, such as, electrodialysis for desalination and reverse electrodialysis for sustainable power generation. However, a tradeoff between conductivity and permselectivity constrains the efficiency of IEM-based technologies. The incorporation of rationally functionalized 1-dimensional nanomaterials as fillers into the polymer matrix offers opportunities to depart from this tradeoff. In this study, we develop nanocomposite cation exchange membranes by incorporating sulfonic acid-functionalized carbon nanotubes, sCNTs, in sulfonated poly(2,6-dimethyl-1,4-phenyleneoxide) polymer matrix. The fabricated nanocomposite IEMs exhibit improved conductivity while maintaining permselectivity. Intrinsic resistivity, the reciprocal of conductivity, is lowered with greater blending of sCNTs fillers, decreasing by approximately $25 \%$ with $20 \mathrm{w} / \mathrm{w} \%$ incorporation of sCNTs, while permselectivity is effectively unchanged across the different degrees of sCNT incorporation (within $2 \%$ variation). Compared with pristine membranes, the conductivity-permselectivity tradeoff line of the fabricated nanocomposite membranes is advantageously advanced, thus improving overall performance. Further characterization and analysis show a percolating network of carbon nanotubes is achieved in the polymer matrix with $10 \mathrm{w} / \mathrm{w} \% \mathrm{sCNTs}$. We posit that the improved effective ionic conductivity is attributed to the interconnected sCNT network reducing the tortuosity of the ion transport path. This study demonstrates the promise of percolating 1D nanomaterial networks to potentially advance the conductivity-permselectivity tradeoff governing conventional IEMs.
\end{abstract}

\section{Introduction}

Ion-exchange membranes (IEMs) are polymeric films with charged moieties, which allow the selective transport of oppositely-charged counterions and retention of like-charged co-ions and electrically neutral species [1]. IEMs are widely utilized in environmental and energy applications [2-5], such as desalination, wastewater treatment, energy harvesting from salinity gradient, and fuel cells. A crucial limitation of IEM-based processes is the low ionic conductivity, $\sigma$, of conventional ion-exchange membranes [6]. The large contribution of the IEMs to the overall resistance of the membrane stack elevates ohmic losses in the circuit that detrimentally lowers the energy efficiency [7-9]. Additionally, the low conductivity also slows process kinetics by diminishing ion flux.

Efforts to enhance the intrinsic conductivity of conventional polymeric IEMs are constrained by a tradeoff relationship between conductivity and permselectivity, $\alpha$, the ability of the membrane to select for counterion passage while repelling co-ions. Empirical evidence from recent studies indicate that an increase in ionic conductivity unavoidably lowers the permselectivity, resulting in a tradeoff between the two key membrane performance parameters (Fig. 1, with vertical axis of selectivity for counterion transport over co-ions presented as $1 /(1-\alpha)$ ) $[5,7,10-13]$. In our previous work, we developed a transport model to show that this conductivity-permselectivity tradeoff relationship intrinsically originates from the structural properties of IEM, specifically the swelling degree (SD), i.e. hydration ability of membrane [14]. A higher SD produces greater conductivity but lowers permselectivity and vice versa. The $\sigma$ - $\alpha$ tradeoff not only curbs the efficiency and kinetics of current IEM process but also narrows the scope of application. For instance, electrodialysis desalination is confined to the lower salinities of brackish water as higher electrolyte concentrations compromise permselectivity [14].

The conventional approach to attain more conductive and selective IEMs is to raise ion exchange capacity (IEC) $[6,15]$, the number of fixed

\footnotetext{
* Corresponding author. Department of Earth and Environmental Engineering, Columbia University, New York, 10027-6623, United States.

E-mail address: n.y.yip@columbia.edu (N.Y. Yip).
} 


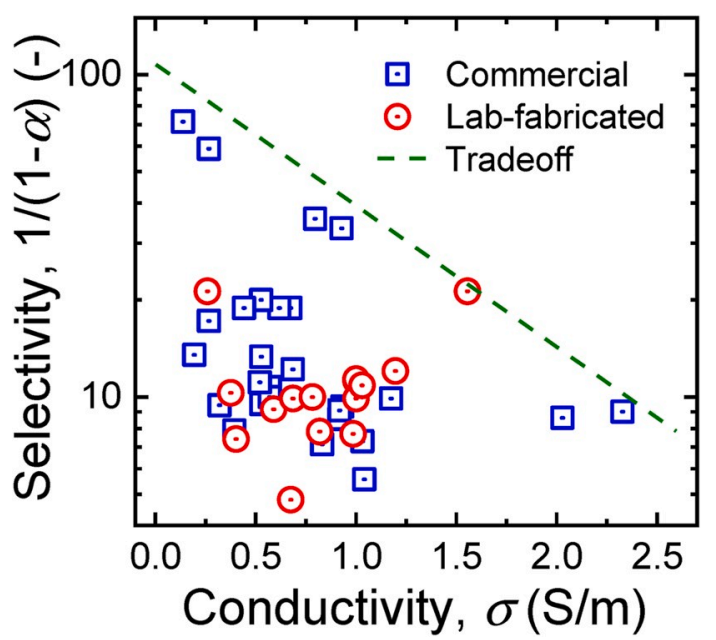

Fig. 1. Selectivity of IEMs reported in literature, presented as $1 /(1-\alpha)$, and the corresponding conductivity, $\sigma$. Blue square and red circle symbols denote commercially-available and laboratory-fabricated IEMs, respectively [5,7, 10-13]. The negative slope of the green line represents the empirical tradeoff between selectivity and conductivity of ion-exchange membranes, where an increase in conductivity is accompanied by a decrease in permselectivity. (For interpretation of the references to colour in this figure legend, the reader is referred to the Web version of this article.)

charges per unit weight of dry membrane. However, raising IEC also incidentally increases membrane swelling degree and dilutes the effective charge density, the number of fixed charges normalized by total volume of the water-swollen IEM. More importantly, the upper limit of achievable IEC is ultimately capped by functionalization chemistry. In addition, a significant portion of the functional groups will not be dissociated at high fixed charge concentrations, i.e., some of the chemical moieties remain neutrally charged [16]. Other approaches to improve $\sigma$ or $\alpha$, such as, using different functional groups [17], blending polymers [18,19], and altering cross-linkage [20,21], generally tune IEM performance via the mechanisms of increasing the effective fixed charge density or controlling the swelling degree to modify free volume in the membrane matrix for ion diffusion. The development of innovative membrane fabrication strategies not based on the traditional mechanisms can potentially yield better IEMs that break away from the current conductivity-permselectivity tradeoff.

Nanomaterials have been utilized in membrane fabrication to augment fluxes, enhance selectivity, and promote mechanical and chemical stabilities [22,23]. Incorporation of nanomaterial filler into the polymer matrix can yield nanocomposite membranes with improved performance, due to the synergism between polymer and nanomaterial. For instance, because of the large aspect ratio and nanoscale dimensions, 1-dimensional nanomaterials, such as, nanotubes and nanowires, can achieve several orders of magnitude greater dispersity than micro-scale fillers and 2- and 3-dimensional nanomaterials [24]. This unique high dispersity feature of 1D nanomaterials can be thoughtfully utilized. For example, when the filler content of composites exceeds a critical value, an interconnected three-dimensional network of the fillers within the matrix is formed, with the critical filler concentration termed percolation threshold [25-27]. For nanotubes with aspect ratio of 300-1,000, the percolation threshold is very low - typically below $1 \mathrm{w} / \mathrm{w} \%$ relative to polymer matrix [24-26]. Carbon nanotubes (CNTs) have been used to form percolating network in polymer composites $[25,28]$. Previous work incorporated CNTs in polymer-electrolyte-based IEMs for fuel cell application to increase proton or hydroxide conductivity while suppressing undesired fuel transport [29-31]. Other studies mixed functionalized CNTs in polyvinylchloride polymer to fabricate "mixed matrix" heterogeneous IEMs, introducing charged groups to reduce the membrane resistance in aqueous solution $[32,33]$. However, the role of filler percolation was not specifically studied and, thus, the potential to advance conductivity-permselectivity tradeoff of ion-exchange membranes has not been fully explored.

This study presents the development and characterization of a new type of nanocomposite cation exchange membrane (CEM). The nanocomposite CEMs were fabricated by incorporating sulfonic acidfunctionalized carbon nanotubes (sCNTs) in sulfonated poly(2,6dimethyl-1,4-phenyleneoxide) (sPPO) polymer matrix, employing the solvent evaporation method. The influence of swelling degree (SD) on the resistivity of fabricated membranes was evaluated by thoughtful control of the heating duration during fabrication to achieve different extents of membrane hydration. The impacts of sCNT loading on the intrinsic resistivity and permselectivity of nanocomposite membranes were then investigated, and the conductivity-permselectivity tradeoff of nanocomposite IEMs was compared to control membranes without sCNT addition. Electrochemical impedance spectroscopy (EIS) analysis of the dry membrane resistance and scanning electron microscopy (SEM) were employed to examine the network of 1-dimensional nanomaterial within the membrane. This work investigates the potential of rational nanomaterials utilization to advance the conductivity-permselectivity tradeoff governing conventional IEMs.

\section{Materials and methods}

\subsection{Materials and chemicals}

Pristine multiwalled carbon nanotubes, CNTs, (20-30 nm outer diameter, 5-10 nm inner diameter, 10-30 $\mu$ m length, 95\% purity) were purchased from Cheap Tubes Inc. (Cambridgeport, VT) and used as received. Concentrated sulfuric acid $\left(\mathrm{H}_{2} \mathrm{SO}_{4}, 98 \%\right)$, acetic anhydride, methanol, sodium hydroxide, chloroform, dimethyl sulfoxide (DMSO), and sodium chloride were acquired from Fisher Scientific (Pittsburgh, PA), whereas chlorosulfonic acid was procured from Sigma-Aldrich (St. Louis, MO). All chemicals are reagent grade and used as received. Poly (2,6-dimethyl-1,4-phenylene oxide) (PPO) was acquired from SigmaAldrich and used as received. Indium foil was obtained from Alfa Aesar (Ward Hill, MA). Deionized (DI) water was purified with a Milli-Q system (Millipore Co., Burlington, MA). Polytetrafluoroethylene (PTFE) membrane disc filters $(0.2 \mu \mathrm{m}$ pore size, $47 \mathrm{~mm}$ diameter $)$ were acquired from Fisher Scientific.

\subsection{Nanocomposite ion-exchange membrane fabrication}

\subsubsection{Sulfonation and characterization of carbon nanotubes}

Sulfonated nanomaterials have been incorporated into polymeric matrices for ion- and proton exchange membranes [34-41]. Sulfonation of the multiwalled CNTs using acetyl sulfuric acid was adopted from a previous study [42]. $20 \mathrm{~mL}$ concentrated sulfuric acid was slowly added to $40 \mathrm{~mL}$ acetic anhydride placed in an ice-water bath and vigorously stirred for $30 \mathrm{~min}$. $40 \mathrm{mg}$ pristine CNTs (pCNTs) were added to the mixture and sonicated for $30 \mathrm{~min}$ in ice-water bath. The mixture was then stirred at $80^{\circ} \mathrm{C}$ for $24 \mathrm{~h}$. The resulting product was diluted by 200 mL DI water, vacuum-filtered with PTFE membrane, and washed by DI water and methanol for three times each. The sulfonated CNTs (sCNTs), depicted in Fig. 2A, were then dried in a vacuum oven at $80^{\circ} \mathrm{C}$ for $12 \mathrm{~h}$. To assess the degree of CNT sulfonation, X-ray photoelectron spectroscopy, XPS (Phi 5500 XPS, PerkinElmer, Inc., Waltham, MA) was employed to characterize the chemical composition of pCNTs and sCNTs. Mg was used as the X-ray source with a work function of $4.5 \mathrm{eV}$, and AugerScan software was used for data collection, curve fitting, and quantitative analysis.

\subsubsection{Sulfonation of $P P O$ polymer}

Poly(2,6-dimethyl-1,4-phenylene oxide) (PPO) was used as the backbone polymer of the IEMs in this study, with sulfonate as the negatively charged functional groups (Fig. 2B). The chemistry to 


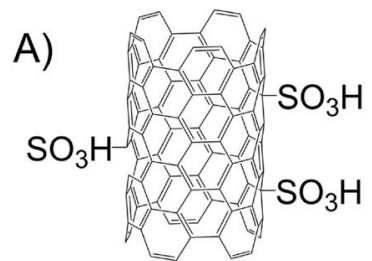

B)

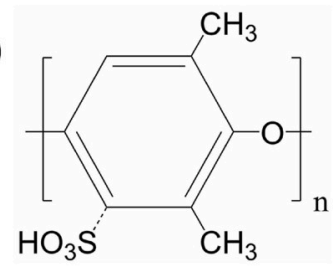

Fig. 2. Structural formulae of A) sulfonated carbon nanotube (sCNT) and B) sulfonated poly( $p$-phenylene oxide) (sPPO). The sCNTs used for membrane fabrication are multiwalled carbon nanotubes, but inner layers of CNTs are not illustrated here for simplicity of presentation.

functionalize PPO with different moieties is facile and the polymer has been previously used to fabricate IEMs [43-45]. To sulfonate PPO, a chlorosulfonic acid sulfonation method was adopted [46]. $6 \mathrm{w} / \mathrm{w} \%$ PPO was dissolved in chloroform by mechanical mixing for $30 \mathrm{~min}$. Then 8 $\mathrm{w} / \mathrm{w} \%$ chlorosulfonic acid solution (chloroform as solvent) was slowly added to the PPO solution over $30 \mathrm{~min}$ under vigorous stirring at room temperature, precipitating the sulfonated PPO. The volume ratio of PPO to chlorosulfonic acid solution was 1:1 to achieve the desired degree of functionalization. Sulfonated PPO (sPPO) polymer was then soaked and washed by DI water, and subsequently dissolved in methanol. The sPPO methanol solution was evaporated in a glass tray for $24 \mathrm{~h}$ to form a layer of thin polymer film. To remove residual acid, the thin film was shredded into small pieces and washed with DI water multiple times until $\mathrm{pH}$ of the rinse water was higher than 4 . After washing, the polymer thin film was dried at room temperature for at least two days.

\subsubsection{Membrane casting}

Sulfonated PPO polymer was dissolved in DMSO to obtain $19 \mathrm{w} / \mathrm{w} \%$ casting solutions. Then, sCNTs were mixed into the DMSO solution to achieve $0-20 \mathrm{w} / \mathrm{w} \%$ relative to SPPO. The sCNT-dispersed solution was immersed in an ultrasonic bath for $40 \mathrm{~min}$ before casting. Solvent evaporation method was employed for IEM fabrication [1]. The solution was cast onto a glass sheet using a casting knife with gate height set at $\approx 1.0 \mathrm{~mm}$. The casted film was dried in a vacuum oven at $80^{\circ} \mathrm{C}$ for $24 \mathrm{~h}$ to remove residual solvents. To obtain membranes with different swelling degree, the samples were further heated at $95{ }^{\circ} \mathrm{C}$ for between 24 and 48 h. Heat-induced self-condensation between sulfonic groups on sPPOs and sCNTs links two chains with $-\mathrm{SO}_{2}-$ and hence eliminates a portion of $-\mathrm{SO}_{3} \mathrm{H}$ functional groups [47]. The loss of sulfonic groups lowers the density of fixed $-\mathrm{SO}_{3} \mathrm{H}$ functional groups and reduces the membrane hydrophilicity [1]. Additionally, the increased crosslinking in the polymer matrix confines the volume of water sorption [48]. These two effects reduce hydration ability of the IEMs.

\subsection{Membrane characterization}

\subsubsection{Swelling degree}

Swelling degree (SD) defines the ability of the IEM matrix to absorb water. The membrane was soaked in DI water for $24 \mathrm{~h}$. Excess water on the sample was carefully removed and the wet weight $\left(W_{\text {wet }}\right)$ was recorded. The dry weight of the membrane $\left(W_{\text {dry }}\right)$ was determined after drying for $24 \mathrm{~h}$ in a vacuum oven at $60{ }^{\circ} \mathrm{C}$. SD is calculated using [49]:

$\mathrm{SD}=\frac{W_{\text {wet }}-W_{\text {dry }}}{W_{\text {wet }}} \times 100 \%$

\subsubsection{Ion exchange capacity}

Ion-exchange capacity (IEC) refers to the specific capacity of the IEM to hold exchangeable counterions and is generally expressed as the number of fixed charges per unit weight of dry membrane [1]. Acidity of the protonated membrane sample was used to determine the IEC of cation exchange membranes (CEMs) fabricated in this study [49]. To protonate all the functional groups, the membrane was immersed in $1 \mathrm{M}$
$\mathrm{HCl}$ solution for $24 \mathrm{~h}$ and washed quickly with DI water. The sample was then soaked in $50 \mathrm{~mL}$ of $1 \mathrm{M} \mathrm{NaCl}$ aqueous solution for $24 \mathrm{~h}$, exchanging $\mathrm{H}^{+}$within the IEM matrix with $\mathrm{Na}^{+} .25 \mathrm{~mL}$ of the soaking solution was titrated with $0.01 \mathrm{M} \mathrm{NaOH}$ using phenolphthalein as indicator. The membrane was dried using the procedure described earlier and the weight, $W_{\text {dry }}$, was recorded. IEC is calculated with

$\mathrm{IEC}=\frac{2 C_{\mathrm{NaOH}} V_{\mathrm{NaOH}}}{W_{\mathrm{dry}}}$

where $C_{\mathrm{NaOH}}$ and $V_{\mathrm{NaOH}}$ are concentration and volume of the titrated $\mathrm{NaOH}$ solution, respectively. Coefficient of two denotes the volume ratio of initial $\mathrm{NaCl}$ soaking solution $(50 \mathrm{~mL})$ to titrated sample $(25 \mathrm{~mL})$.

\subsubsection{Morphology}

To prepare membrane for imaging that are representative of the swollen state, the fabricated IEM coupons were soaked in $1 \mathrm{M} \mathrm{NaCl}$ for at least $24 \mathrm{~h}$. The water-swollen IEMs were then dipped into liquid nitrogen for $\approx 40 \mathrm{~s}$. Using tweezers, the membranes were carefully snapped in half to expose the cross-section. The samples were mounted onto specimen holders with carbon tape. To eliminate undesired static electric charge effect, $\approx 10 \mathrm{~nm}$ Au coating was applied before imaging. The samples were analyzed with scanning electron microscopy, SEM (SIGMA VP, Carl Zeiss, Germany).

Electrochemical impedance spectroscopy (EIS) with an electrochemical workstation (Interface 1010E, Gamry Instruments, Warminster, PA) was utilized to characterize the membranes at dry state [50, 51]. Newly fabricated membranes were soaked in $1 \mathrm{M} \mathrm{NaCl}$ solution overnight then dehydrated in vacuum oven for $12 \mathrm{~h}$. The dry membranes were sandwiched between Au-coated coin electrodes ( $16 \mathrm{~mm}$ diameter) and scanned with alternating current over the frequency range from 1 $\mathrm{Hz}$ to $1 \mathrm{MHz}$.

\subsubsection{Area specific resistance and resistivity}

An electrochemical test setup based on a four-electrode cell system was used for resistance measurements [1]. The two chambers of the cell ( $\approx 16 \mathrm{~mL}$ in each chamber) were separated by a $2 \mathrm{~cm} \times 2 \mathrm{~cm}$ membrane coupon. The terminal working electrodes are Pt-coated Ti mesh $(4 \mathrm{~cm} \times$ $4 \mathrm{~cm}$ ). Two $\mathrm{Ag} / \mathrm{AgCl}$ reference electrodes (BASi RE-5B, Bioanalytical Systems, Inc., West Lafayette, IN), positioned $7 \mathrm{~mm}$ apart and straddling the IEM, measures the potential difference across.

Membrane resistance was measured using an electrochemical workstation (Interface 1010E). The chambers of the test cell were filled with $1 \mathrm{M} \mathrm{NaCl}$ electrolyte solution. A scanning direct current was applied, from 1 to $10 \mathrm{~mA}$ (i.e., current density of $0.25-2.5 \mathrm{~mA} / \mathrm{cm}^{2}$ ) in increments of $1 \mathrm{~mA}$, and voltage drop across the membrane was recorded. Each current step was maintained for $10 \mathrm{~s}$ during which the voltage drop was sampled 10 times. The slope of voltage drop as a function of current density $\left(\mathrm{d} V_{\mathrm{m}} / \mathrm{d} i\right)$, after subtracting the blank test reading $\left(\mathrm{d} V_{\text {blank }} / \mathrm{d} i\right)$, i.e., recorded resistance without IEM, yields the area specific resistance (ASR) of the membrane:

$\operatorname{ASR}=\frac{\mathrm{d} V_{\mathrm{m}}}{\mathrm{d} i}-\frac{\mathrm{d} V_{\text {blank }}}{\mathrm{d} i}$

Wet thickness of the membranes, $l$, was determined using a digital micrometer. The area specific resistance normalized by wet thickness returns the membrane intrinsic resistivity, $\rho$, which is the reciprocal of conductivity, $\sigma$, i.e., $\rho=\mathrm{ASR} / l=\sigma^{-1}$.

\subsubsection{Permselectivity}

Permselectivity, $\alpha$, describes the selectivity of the IEM for counterion transport over co-ions. The same test cell and electrochemical workstation employed for resistance characterization were used for permselectivity measurements. The static method was adopted [1], which utilizes the potential difference across the membrane separating two solutions of different concentrations to determine the apparent 
permselectivity, $\alpha$. In this study, 0.1 and $0.5 \mathrm{M} \mathrm{NaCl}$ solutions were circulated on opposite sides of the IEM. The potential difference between the two $\mathrm{Ag} / \mathrm{AgCl}$ reference electrodes, $\psi$ measured, was averaged over 15 min after it had stabilized (fluctuations were within $0.1 \mathrm{mV}$ over $5 \mathrm{~min}$ ) [52-54]. Offset potential between the reference electrodes, $\psi_{\text {offset, }}$ was measured in $0.5 \mathrm{M} \mathrm{NaCl}$ solution with the same stabilization criterion. Junction potential difference between the two reference electrodes, $\psi_{\text {junction, }}$ across 0.5 and $0.1 \mathrm{M} \mathrm{NaCl}$ solutions was estimated as $7.9 \mathrm{mV}$ using the activity-corrected form of the Henderson equation [52]. Theoretical Nernst potential ( $\psi_{\text {theoretical }}$ ) between 0.5 and $0.1 \mathrm{M} \mathrm{NaCl}$ solutions was calculated as $37.9 \mathrm{mV}$ [11]. Solution-phase transport numbers for counterion, $t_{\mathrm{ct}}$, and co-ion, $t_{\mathrm{co}}$, which are $\mathrm{Na}^{+}$and $\mathrm{Cl}^{-}$in this study, were calculated as 0.396 and 0.604 , respectively [55]. Apparent permselectivity is determined by [56]:

$\alpha=\frac{\frac{\psi_{\text {measured }}-\psi_{\text {offset }}-\psi_{\text {junction }}}{\psi_{\text {theorectical }}}+1-2 t_{c t}}{2 t_{c o}}$

\section{Results and discussion}

\subsection{Characteristics of sulfonated CNTs}

Multiwalled carbon nanotubes were functionalized using acetyl sulfuric acid, decorating the outer wall of the nanotubes with sulfonic acid functional groups. XPS was employed to analyze the surface elemental compositions of the pristine and sulfonated carbon nanotube, pCNT and sCNT, respectively, and the survey spectra are presented in Fig. 3. Both spectra of pCNT and sCNT show a sharp carbon peak at binding energy of $285 \mathrm{eV}$ [57], as expected for the carbon-based nanomaterial (labeled C1s in Fig. 3A). The peaks at around $450 \mathrm{eV}$ are the signal of the indium foil substrate [57]. Around the characteristic binding energy of oxygen (O1s, $\approx 532 \mathrm{eV}$ ) [57], both spectra display a peak for oxygen components: pCNT presents a weak peak for $\mathrm{C}-\mathrm{O}$ and $\mathrm{C}=\mathrm{O}$ defects on carbon nanotubes, whereas the sCNT spectrum exhibits a stronger signal due to additional $\mathrm{S}=\mathrm{O}$ and $\mathrm{S}-\mathrm{OH}$ functional groups produced in the sulfonation reaction.

The major difference between the spectra of pCNT and sCNT lies in the sulfur peak region $(\approx 168 \mathrm{eV})$ [57]. In the S2p spectrum of pCNT (Fig. 3B), no sulfur signal can be identified, i.e., as expected, sulfur is not present in pCNT. In contrast, a sulfur peak is observed in the S2p spectrum of sCNT and can be decomposed into $\mathrm{S} 2 \mathrm{p}_{1 / 2}$ at $169.48 \mathrm{eV}$ and S2 $\mathrm{p}_{3 / 2}$ at $167.71 \mathrm{eV}$ (Fig. 3C) [57]. Specifically, the binding energy of the sulfur peak matches the features of sulfate (168-171 eV) [57], thus validating the presence of sulfonic acid groups on the carbon nanotubes.

Quantitative analysis of surface elemental composition was carried out by combining multiplex scan results with the relative sensitivity factors of each element [57]. The atom ratio and weight percentage of the elements for pCNT and sCNT are summarized in Table 1 . The $\mathrm{O}$ and $\mathrm{S}$ ratios of peak area to sensitivity factor is normalized by $\mathrm{C}$ to determine the atom ratios. The atom ratio is converted to weight percentage by factoring in the atomic weight of each element. Dividing the mass fraction of $S$ in the sCNT by the molecular weight of $S(32 \mathrm{~g} / \mathrm{mol})$ yields the effective IEC of the sulfonated carbon nanotubes. Mass concentration of sulfur in SCNT is around 3.5\%, whereas no sulfur was detected in the pCNT. The sCNTs have an equivalent $-\mathrm{SO}_{3} \mathrm{H}$ ion-exchange capacity of $1.1 \mathrm{meq} / \mathrm{g}$, comparable to the IEC of commonly used ion-exchange membranes (1.0-1.6 meq/g) [15]. The results verify that the CNTs were effectively sulfonated for subsequent use in the fabrication of nanocomposite IEMs.

To investigate the impact of functionalization on CNT stability in casting solution, pCNTs and sCNTs were sonicated in casting solvent of DMSO for 3 min to obtain well-dispersed suspensions (Fig. 4). After $10 \mathrm{~h}$, pCNTs aggregated and settled to the bottom, while sCNTs remained stable in DMSO for more than 3 months. This result confirms that sulfonation enhances the dispersibility of CNTs in DMSO, which can facilitate the incorporation of nanotubes into the polymer matrix of the
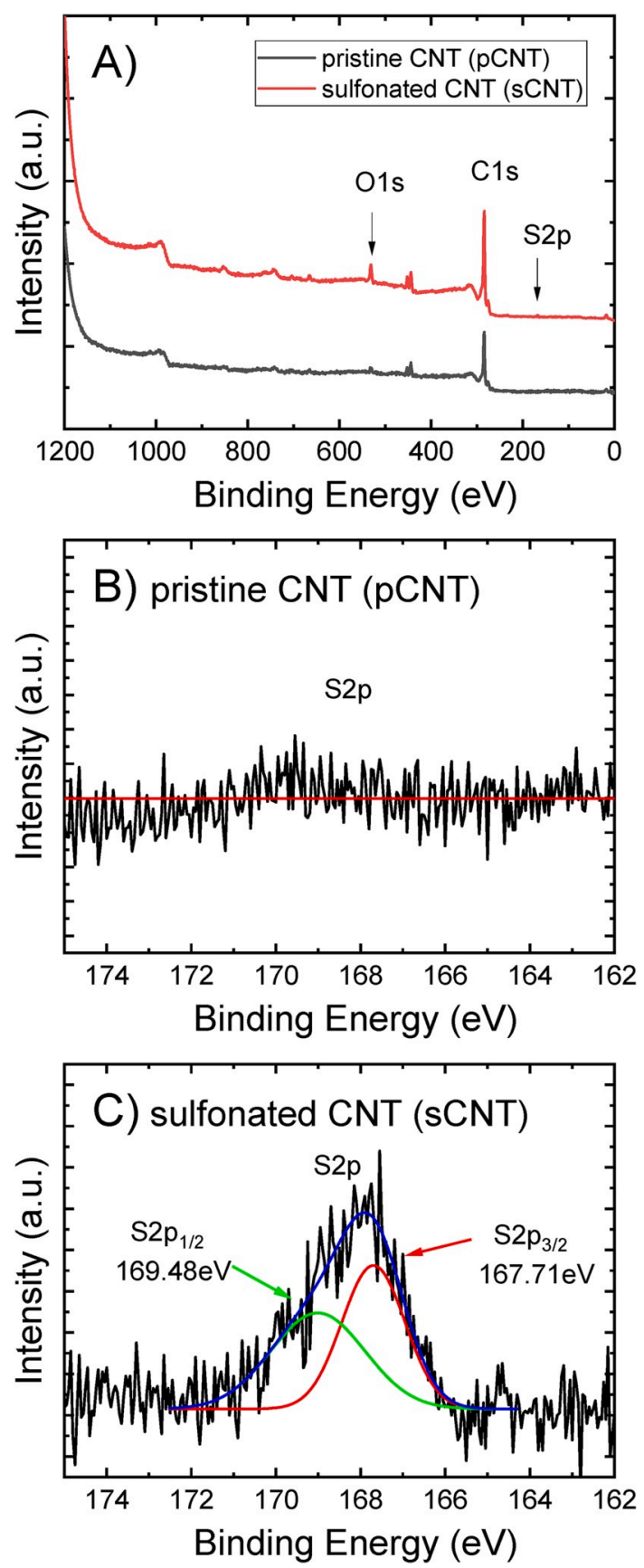

Fig. 3. XPS spectra of pristine and sulfonated carbon nanotube, pCNT and sCNT, respectively: A) full XPS spectra survey, B) S signal of non-sulfonated pCNT, and C) S peak of sCNT.

Table 1

XPS atomic analysis of pristine and sulfonated carbon nanotube, pCNT and sCNT, respectively.

\begin{tabular}{|c|c|c|c|c|c|c|}
\hline & \multicolumn{3}{|c|}{ Peak area } & \multirow{2}{*}{$\begin{array}{l}\text { Atom } \\
\text { ratio (C: } \\
\mathrm{O}: \mathrm{S})\end{array}$} & \multirow{2}{*}{$\begin{array}{c}\text { Weight } \\
\text { percentage (C\%: } \\
\text { O\%: S\%) }\end{array}$} & \multirow{2}{*}{$\begin{array}{c}\text { IEC of } \\
-\mathrm{SO}_{3} \mathrm{H} \\
\text { (meq/g) }\end{array}$} \\
\hline & $\mathrm{C}$ & $\mathrm{O}$ & S & & & \\
\hline pCNT & 13,997 & 2,557 & - & $\begin{array}{c}1: 0.076: \\
0\end{array}$ & $90.8: 9.8: 0$ & - \\
\hline sCNT & 12,768 & 4,186 & 471 & $\begin{array}{c}1: 0.136: \\
0.016\end{array}$ & $81.7: 14.8: 3.5$ & 1.1 \\
\hline
\end{tabular}



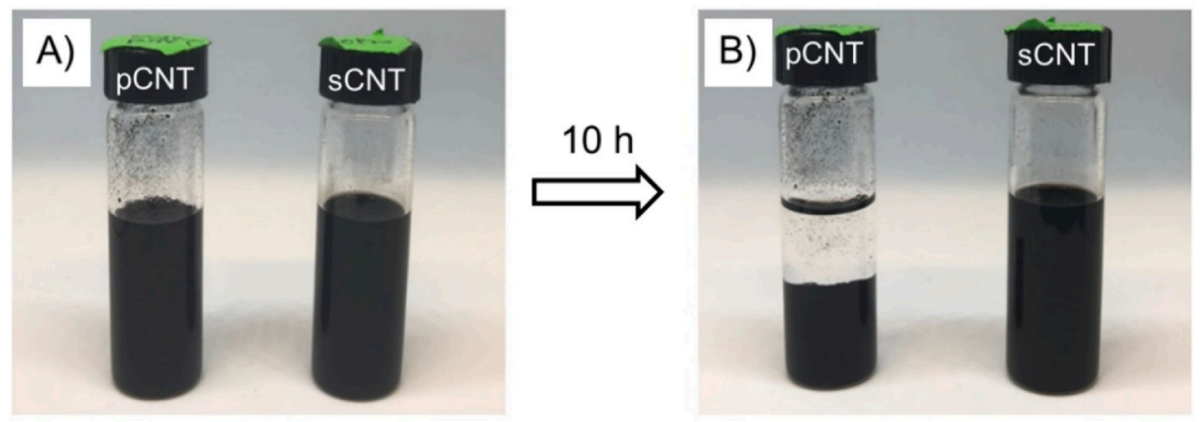

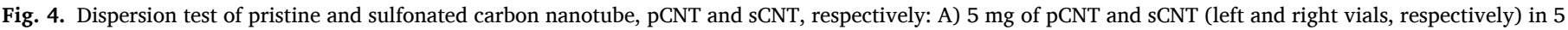

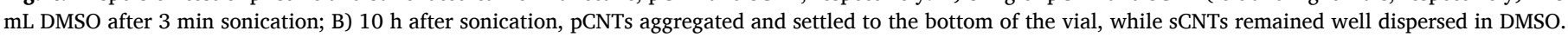

membrane.

\subsection{Resistivity of fabricated IEMs with different $S D$}

Nanocomposite IEMs with $20 \mathrm{w} / \mathrm{w} \%$ sCNT were prepared with different heating durations during fabrication and the resultant intrinsic resistivity, $\rho$, and swelling degree, SD, are presented in Fig. 5, together with the control of sPPO-only membranes, i.e., no sCNTs. SD describes the ability of the polymer matrix to sorb water and is an important structural parameter influencing the ionic resistivity and permselectivity of IEMs. Greater membrane hydration, that is, higher SD, beneficially lowers tortuosity and increases free volume for diffusion, thus facilitating ion transport, i.e., lower $\rho$ [14]. This negative correlation between $\rho$ and SD has been reported by empirical studies $[7,58,59]$.

By purposefully varying the heating time at $95^{\circ} \mathrm{C}$ from 24 to $48 \mathrm{~h}$, membranes with different SD were obtained. Swelling degree of the control IEMs and fabricated sCNT nanocomposites varies from 19.5 to $63.9 \%$ and $23.4-57.9 \%$, respectively, and are in the range of current commercial IEMs (8-56\%) [60]. Correspondingly to the spread of SD, IEC of pristine membranes ranges from $1.21-1.90 \mathrm{meq} / \mathrm{g}$, whereas 20 $\mathrm{w} / \mathrm{w} \% \mathrm{sCNT}$ nanocomposite membranes exhibit relatively lower IEC of $1.00-1.67 \mathrm{meq} / \mathrm{g}$. When SD is reduced, the resistivity of nanocomposite IEMs increased from 103 to $333 \Omega \mathrm{cm}$, whereas for sPPO-only

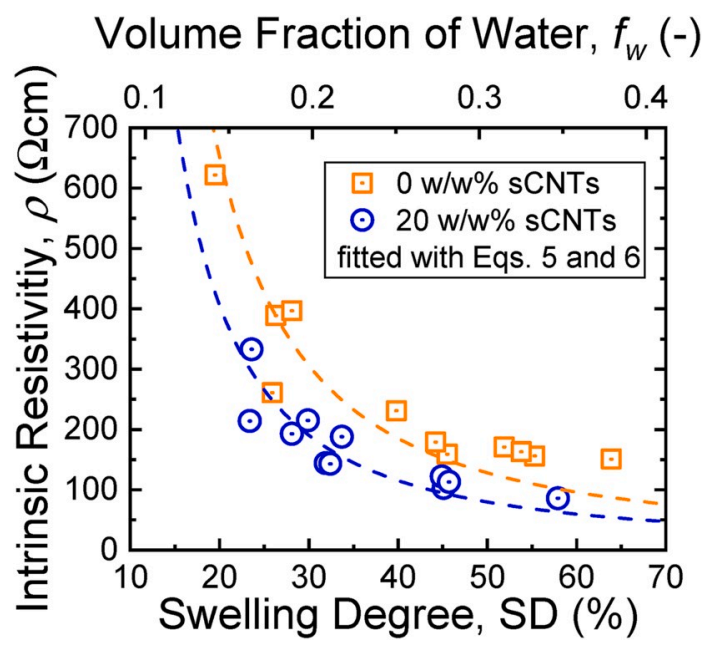

Fig. 5. Intrinsic resistivity, $\rho$, and the corresponding swelling degree, $\mathrm{SD}$, for sCNT-incorporated membranes and sPPO-only membranes as control (blue circle and orange square symbols, respectively). The equivalent volume fraction of water of the membranes, $f_{\mathrm{w}}$, is indicated on the top horizontal axis. The dashed lines denote the fitted $\rho$-SD relationship based on Eqs. (5) and (6), where fitting parameter $\lambda$ is 3.16 and 5.07 for sCNT nanocomposite IEMs and control membranes, respectively. (For interpretation of the references to colour in this figure legend, the reader is referred to the Web version of this article.) membranes, resistivity rose from 86 to $622 \Omega \mathrm{cm}$. As depicted in Fig. 5, the intrinsic resistivities of both CNT-mixed and control IEMs are negatively correlated with $\mathrm{SD}$, in agreement with the theoretical framework for IEM transport [14]. The rise in membrane resistivity is especially pronounced as SD falls below $\approx 30 \%$. Comparing the $\rho$ and SD data shows that incorporation of sCNTs leads to reduced membrane resistivity across the SD range investigated (blue circle symbols are generally to the lower left of orange square symbols). For instance, at swelling degree $\approx 45 \%$, membranes without sCNTs exhibit an average resistivity of $169 \Omega \mathrm{cm}$, while addition of $20 \mathrm{w} / \mathrm{w} \%$ sCNT to the membrane lowered $\rho$ to $112 \Omega \mathrm{cm}$ (33.7\% decrease). This resistivity reduction of the nanocomposite IEMs is more amplified at lower swelling degrees, especially when SD is less than $\approx 20 \%$.

The inverse relationship between SD and $\rho$ can be modeled based on ion transport analysis presented in our previous study [14]:

$$
\begin{gathered}
f_{\mathrm{w}}=\frac{\mathrm{SD}}{\mathrm{SD}+\phi_{\mathrm{p}}^{-1}} \\
\rho=\lambda \frac{\left(2-f_{\mathrm{w}}\right)^{2}}{f_{\mathrm{w}}^{2}}
\end{gathered}
$$

where $f_{\mathrm{w}}$ is the volume fraction of water in ion-exchange membrane and is related to SD through dry polymer density, $\phi_{\mathrm{p}}$ (Eq. (5)). The influences of fixed charge density, ion diffusivity, and counterion condensation are aggregated into fitting parameter, $\lambda$, to enable simplification of the analysis by isolating the confounding effect of $f_{\mathrm{w}}$ (or, equivalently, SD) without needing to separately quantify the individual influence of the different phenomena $[14,61]$. The experimental data of resistivity and SD for the nanocomposite and control membranes are fitted to Eqs. (5) and (6), shown in Fig. 5 as blue and orange dashed lines, respectively. The fitted $\rho$-SD trendlines enable direct quantitative comparison of IEM resistivity by excluding the free volume effect. Down and leftwards shift of the $\rho$-SD curve after sCNTs incorporation is clearly evident. The fitting parameter $\lambda$ for sCNT nanocomposite membranes and control membranes are 3.16 and 5.07, respectively, indicating that the blending of 20 w/w $\%$ sCNT favorably lowers resistivity by $\approx 38 \%$ for the same $f_{\mathrm{w}}$. Therefore, the introduction of rationally functionalized carbon nanotubes as fillers to the polymer matrix is a swelling degree-independent approach to advantageously improve IEM ionic conductivity.

\subsection{Nanocomposite IEM resistivity drops with greater loading of sCNT}

The impacts of different sCNT loading on membrane structural properties and performance parameters were further investigated. Weight ratio of sCNT to composite, i.e., polymer matrix and sCNTs, was tuned from 0 to $20 \mathrm{w} / \mathrm{w} \%$ using the membrane fabrication protocol described in Section 2.2, except the heating time at $95{ }^{\circ} \mathrm{C}$ is fixed at $24 \mathrm{~h}$ to produce membranes with similar swelling degree. Digital images, structural properties of wet thickness, $l$, ion-exchange capacity, IEC, and 
swelling degree, SD, and electrochemical parameters of area specific resistance, ASR, intrinsic resistivity, $\rho$, and permselectivity, $\alpha$, are presented in Table 2 for the sPPO-only and sCNT-incorporated membranes. Experimental variations of IEC and SD between the membranes with different SCNT loadings are largely attributed to the inherent capricious tendency of the condensation reaction of sulfonic groups during membrane fabrication.

The polymeric membranes without CNT, i.e., controls, are translucent yellowish thin films. Incorporation of sCNTs tinged the membranes black, with increasing CNT loading resulting in progressive intensification of opacity. The uniformity of the $2.0,10$, and $20 \mathrm{w} / \mathrm{w} \%$ sCNT membranes, without aggregated CNT bundles visible, corroborates the excellent dispersion of nanotubes in the membrane matrix. Thickness of membranes at water-swollen state with $0-20 \mathrm{w} / \mathrm{w} \% \mathrm{sCNT}$ loading ranged from 75 to $105 \mu \mathrm{m}$. Ion-exchange capacity, which describes the density of membrane fixed charges, of 1.48-1.84 meq/g were measured for the fabricated IEMs, comparable to the typical range of 1-3 $\mathrm{meq} / \mathrm{g}$ reported in literature for commercial and lab-fabricated IEMs [6]. Generally, IEC drops with the incorporation of sCNTs. This decrease in IEC is attributed to the difference in degree of functionalization between the sulfonated carbon nanotubes and the charged polymer. The equivalent $-\mathrm{SO}_{3} \mathrm{H}$ capacity of the lab-functionalized sCNTs is $\approx 1.1$ $\mathrm{meq} / \mathrm{g}$ (Table 1), whereas IEC of the sPPO polymer matrix is higher at around $1.84 \mathrm{meq} / \mathrm{g}$ (equivalent to IEC of the polymer-only control membranes). Therefore, the mixing of sCNT fillers into the polymer matrix effectively dilutes the density of fixed functional groups and, hence, lowers the overall membrane IEC. Increasing sCNT loading also, in general, reduces the swelling degree of fabricated nanocomposite membranes. Adding sCNTs to the sPPO polymer lessened SD from $53.8 \%$ to $42.3-49.9 \%$, as the nanocomposite membranes are rendered less hydrophilic due to the diminished IEC [1] and membrane hydration capacity is, therefore, depressed.

The influence of sCNT loading on IEM membrane performance was characterized by electrochemical measurements of area specific resistance, ASR, membrane intrinsic resistivity, $\rho$, and apparent permselectivity, $\alpha$ (Table 2). Specifically, intrinsic resistivity, the reciprocal of conductivity, is ASR normalized by $l$ and is, hence, an intensive property of the IEM, i.e., independent of dimensions and amount of material. Therefore, by excluding the confounding factor of inherent experimental variations in membrane thickness, $\rho$ is a more accurate and insightful parameter to quantify the effects of sCNT addition. Intrinsic resistivity and permselectivity data of Table 2 are presented in Fig. 6 as a function of sCNT loading (red square symbols, left vertical axis, and green circle symbols, right vertical axis, respectively).

Fig. 6 shows an overall decreasing trend in intrinsic resistivity, from 163 to $122 \Omega \mathrm{cm}$, of the nanocomposite membranes with increasing amount of sCNT incorporation. Resistivity of the $2.0 \mathrm{w} / \mathrm{w} \%$ sCNT IEMs are only marginally lower than the polymer-only membranes $(-1.2 \%)$. However, a sharp reduction in $\rho$ of $23 \%$ (relative to control) is observed between 2.0 and $10 \mathrm{w} / \mathrm{w} \%$, followed by a further gentle decrease from

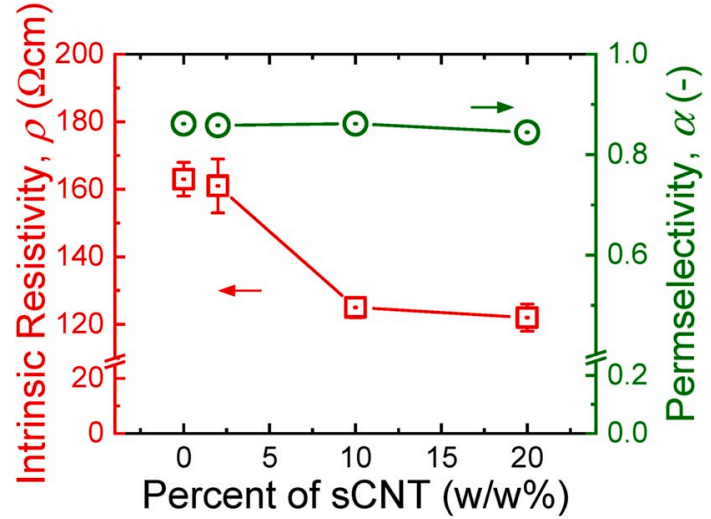

Fig. 6. Intrinsic resistivity, $\rho$ (left vertical axis, red square symbols), and permselectivity, $\alpha$ (right vertical axis, green circle symbols), as a function of weight percent of lab-functionalized sCNTs in IEM. $\rho$ was measured by fourelectrode direct current test, whereas $\alpha$ was characterized by the static method, which utilizes voltage difference across the membrane separating 0.1 $\mathrm{M}$ and $0.5 \mathrm{M} \mathrm{NaCl}$ solutions. (For interpretation of the references to colour in this figure legend, the reader is referred to the Web version of this article.)

10 to $20 \mathrm{w} / \mathrm{w} \%$ ( $-25 \%$ compared to $0 \mathrm{w} / \mathrm{w} \%$ ). The steep drop-off in resistivity between 2.0 and $10 \mathrm{w} / \mathrm{w} \%$ is possibly caused by the formation of a percolating carbon nanotube network $[24,25,62]$, where the CNTs are continuously connected across the membrane thickness. The contiguous network of CNT fillers within the polymer matrix and the percolation threshold of nanotubes loading at which the lattice forms are further discussed in Section 3.5. In contrast to the declining resistivity trend, the membrane apparent permselectivity, $\alpha$, a measure of IEM selectivity for counterion transport over co-ion, is practically preserved across the sCNT loadings investigated (Table 2 and Fig. 6). Compared to the sPPO-only control membranes, $\alpha$ of the nanocomposite IEMs with $2.0-20 \mathrm{w} / \mathrm{w} \%$ differs by $<2.0 \%$, indicating permselectivity of the fabricated membranes is effectively not influenced by the incorporation of sCNTs. Therefore, the blending of sulfonated CNTs filler into the sPPO polymer matrix yields enhanced ionic conductivity without compromising counterion selectivity in the nanocomposite IEMs. The impact of sCNT addition on conductivity-permselectivity tradeoff is further analyzed in Section 3.4.

Mixing the lab-functionalized sCNT into the membrane incidentally lowers SD and IEC (Table 2). The equivalent fixed charge density, which is the concentration of fixed charged moieties in water swollen membrane matrix (calculated as IEC $\times \rho_{\mathrm{w}} / \mathrm{SD}$, where $\rho_{\mathrm{w}}$ is the density of water), of the $20 \mathrm{w} / \mathrm{w} \% \mathrm{sCNT}$ membranes also dropped from 3.42 to $3.30 \mathrm{eq} / \mathrm{L}$ water sorbed, compared to $0 \mathrm{w} / \mathrm{w} \%$ samples. For conventional ion-exchange membranes, the drop in SD and IEC would deleteriously elevate ionic resistivity $[14,15,60]$, because of i) diminished effective ion diffusivity at lower swelling degrees, thus causing an inverse

Table 2

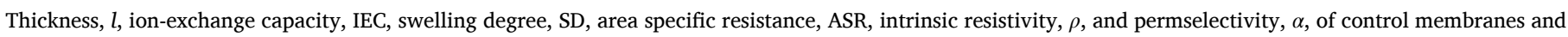
nanocomposites with 2.0-20 w/w\% sCNT loading. Digital images of the membranes are shown in the corresponding columns.

\begin{tabular}{|c|c|c|c|c|}
\hline sCNT & $0 \mathrm{w} / \mathrm{w} \%$ & $2.0 \mathrm{w} / \mathrm{w} \%$ & $10 \mathrm{w} / \mathrm{w} \%$ & $20 \mathrm{w} / \mathrm{w} \%$ \\
\hline Thickness, $l[\mu \mathrm{m}]$ & $86 \pm 7$ & $75 \pm 8$ & $105 \pm 3$ & $98 \pm 5$ \\
\hline IEC $[\mathrm{meq} / \mathrm{g}]$ & 1.84 & 1.54 & 1.66 & 1.48 \\
\hline SD [\%] & 53.8 & 42.3 & 49.9 & 44.9 \\
\hline ASR $\left[\Omega \mathrm{cm}^{2}\right]$ & $1.39 \pm 0.04$ & $1.21 \pm 0.06$ & $1.31 \pm 0.03$ & $1.19 \pm 0.04$ \\
\hline$\rho[\Omega \mathrm{cm}]$ & $163 \pm 5$ & $161 \pm 8$ & $125 \pm 3$ & $122 \pm 4$ \\
\hline$\alpha[-]$ & $\underline{0.862}$ & $\underline{0.858}$ & $\underline{0.862}$ & $\underline{0.845}$ \\
\hline
\end{tabular}


relationship between $\rho$ and SD (explained earlier in Section 3.2) [14,59] and ii) the fall in IEC, and equivalently fixed charge density, reduces the concentration of mobile counterions inside the membrane, which are the main current carriers, thus resulting in smaller ion fluxes and, correspondingly, larger resistivity [14,60]. Despite the unfavorably decreased SD and IEC of the nanocomposite IEMs, incorporation of sCNT still improved ion permeability and reduced resistivity, signifying the conductivity enhancements are caused by a different mechanism that arises from the presence of 1-dimensional nanomaterial filler in the polymer matrix. Section 3.5 further examines this underlying phenomenon.

\subsection{Incorporation of sCNTs advances the IEM conductivity- permselectivity tradeoff}

High conductivity and high permselectivity are desired in IEMs for fast and efficient charge separations. A previous theoretical study on IEM transport established that conductivity, $\sigma$, can be improved by raising swelling degree but permselectivity, $\alpha$, is detrimentally compromised, i.e., varying SD produces a tradeoff between conductivity and permselectivity [14]. The role of water uptake in IEM $\sigma$ - $\alpha$ tradeoff relationship was also observed by empirical studies [7,58,59]. To investigate the effect of sCNT fillers on the SD-controlled conductivity-permselectivity tradeoff, $20 \mathrm{w} / \mathrm{w} \%$ sCNTs nanocomposites and sPPO-only membranes with different SD were prepared by purposefully altering the fabrication condition of heating duration.

Fig. 7 shows the permselectivity falls as conductivity increases with higher SD for both the sCNT-incorporated and control membranes (blue circle and orange square symbols, respectively). For membranes with sCNT fillers, permselectivity declines from 0.914 to 0.845 , whereas conductivity is elevated from 0.300 to $0.885 \mathrm{~S} / \mathrm{m}$. The same negatively correlated trend is observed for the control samples: $\alpha$ decreases from 0.902 to 0.837 while $\sigma$ increases from 0.252 to $0.662 \mathrm{~S} / \mathrm{m}$. Linear regression on permselectivity and conductivity of nanocomposites and sPPO-only membranes are indicated by blue and orange dashed lines, respectively. Both trendlines exhibit negative slopes with similar gradients of -0.115 and -0.112 for sPPO-only control membranes and nanocomposite IEMs, respectively. As depicted in Fig. 7, integrating sCNT into the polymer matrix of cation exchange membranes shifts the conductivity-permselectivity tradeoff line outwards, to the top right, i.

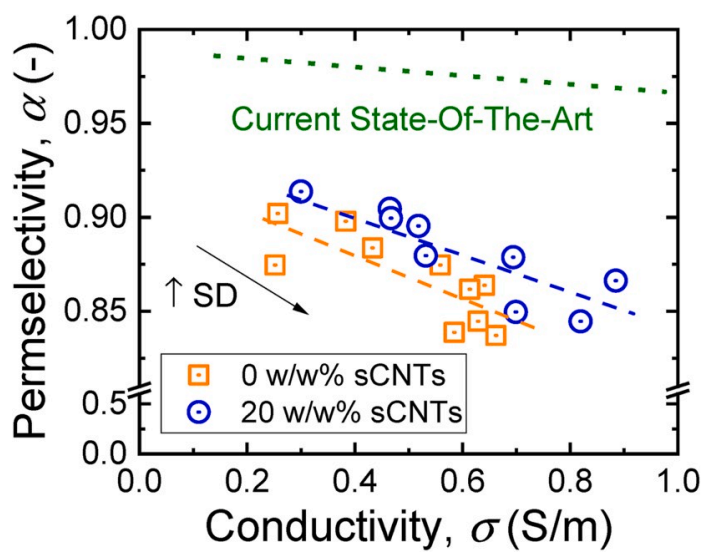

Fig. 7. Permselectivity, $\alpha$, and the corresponding conductivity, $\sigma$, for sCNTincorporated membranes and sPPO-only control membranes (blue circle and orange square symbols, respectively). IEMs with a range of swelling degrees were fabricated to obtain the $\alpha$ and $\sigma$ distribution. Direction of black arrow denotes increasing swelling degree. The dashed lines denote linear regression on the $\alpha-\sigma$ relationship, where slopes of $-0.1 \underline{12}$ and -0.115 were obtained for the fitting of sCNT nanocomposite IEMs and control membranes, respectively. The green dotted line approximately represents the empirical tradeoff between permselectivity and conductivity as presented in Fig. 1. (For interpretation of the references to colour in this figure legend, the reader is referred to the Web version of this article.) e., for the same conductivity, nanocomposite IEMs have higher permselectivity than polymer-only membranes.

The results indicate the incorporation of sCNT can advance the conductivity-permselectivity tradeoff that constrains conventional ionexchange membranes. Unlike the common strategy of introducing more fixed charged functional groups into the membrane polymer matrix to bolster performance, the thoughtful dispersion of sCNT to produce nanocomposite IEMs enhances $\alpha$ and $\sigma$ even though IEC is adversely lowered. This implies that performance improvements are likely due to other structural changes caused by the addition of SCNT fillers, and is further investigated in the next Section.

\subsection{Conductivity improvements are attributed to percolating network of sCNTs in IEM polymer matrix}

SEM micrographs of the sPPO-only membranes (top row, A-C) and nanocomposites with $20 \mathrm{w} / \mathrm{w} \%$ sCNTs (bottom row, D-F) are presented in Fig. 8. Fig. 8A and D show the planar surface, Fig. 8B and E display the IEM cross-sections, and Fig. $8 \mathrm{C}$ and $\mathrm{F}$ are further zoomed-in crosssectional views $(10,000,500$, and 50,000 $\times$ magnification, respectively). Surface of the control sPPO thin film is dense and relatively flat (Fig. 8A). A similar nonporous and smooth morphology is observed in the cross-sectional views of Fig. 8B and C (flakes and small shreds of polymer Fig. $8 \mathrm{~B}$ are artefacts of sample preparation when the membrane is snapped to expose the cross-section). The dense morphology is also observed in the polymer matrix of the $20 \mathrm{w} / \mathrm{w} \%$ sCNT membranes (Fig. 8D-F) but, additionally, CNTs are visible (bright rods and dots as the nanotubes are electrically conducting). The 1D nanomaterials are clearly seen protruding out of the membrane surface and are especially prominent in the cross-sectional micrographs (Fig. 8E and F). The width of the rods observed in the SEM micrographs is measured to be $26.8 \pm$ $2.3 \mathrm{~nm}$ (averaged by 6 readings), corresponding well with the manufacturer's specifications of 20-30 nm outer diameter. The generally even distribution of bright rods and dots further substantiates the uniform dispersion of CNTs within the polymer matrix of the nanocomposite IEMs, consistent with the digital images of Table 2. SEM micrographs of the $10 \mathrm{w} / \mathrm{w} \% \mathrm{sCNT}$ IEMs (results not shown) are visually similar to the $20 \mathrm{w} / \mathrm{w} \%$ samples, albeit with a lower density of nanotubes, i.e., lesser bright rods and dots.

Electrochemical impedance spectroscopy (EIS) tests were conducted on the membranes in dry state to probe the electrical conductivity with different sCNT loading. Changes in the net electrical conductivity measured provide information on the dispersion of electricallyconductive CNT fillers within the relatively insulating polymer matrix $[26,63]$. Fig. 9 shows the through-plane conductivity of membranes (logarithmic scale) as a function of weight percent of sCNT incorporation. Note that EIS conductivity is a measure of the conductivity of electrons across the membrane and is different from the hydrated conductivity (i.e., IEM in aqueous solution) discussed earlier, which represents ionic conductivity in electrolyte solutions. Dry conductivity, $\kappa$, of the pristine polymeric membrane, determined from the fitting of EIS impedance results, is $2.57 \times 10^{-6} \mathrm{~S} / \mathrm{m}$. $\kappa$ initially rises to $8.25 \times 10^{-5}$ $\mathrm{S} / \mathrm{m}$ for the $2.0 \mathrm{w} / \mathrm{w} \%$ sCNT IEM and then increases by almost 4 orders of magnitudes to $5.57 \times 10^{-3} \mathrm{~S} / \mathrm{m}$ for $10 \mathrm{w} / \mathrm{w} \%$ sCNT. Doubling the sCNT loading further to $20 \mathrm{w} / \mathrm{w} \%$ yields a comparatively modest elevation of $\kappa$ to $2.15 \times 10^{-2} \mathrm{~S} / \mathrm{m}$. The dry conductivity trend is in agreement with the percolating network explanation postulated earlier in Section 3.3: when CNT loading increases beyond a certain threshold, the 1D nanotubes form an uninterrupted network that enables the continuous transport of electrons across the membrane thickness through the electron-conducting CNTs [26,63], thus resulting in a drastic increase in electrical conductivity. Increasing the CNT loading beyond the percolating threshold produces a diminishing return in conductivity enhancement $[24,25,62]$. From the data of Figs. 6 and 9, the threshold at which the contiguous lattice of CNT filler is formed is estimated to be between 2.0 and $10 \mathrm{w} / \mathrm{w} \%$. Therefore, the impedance 

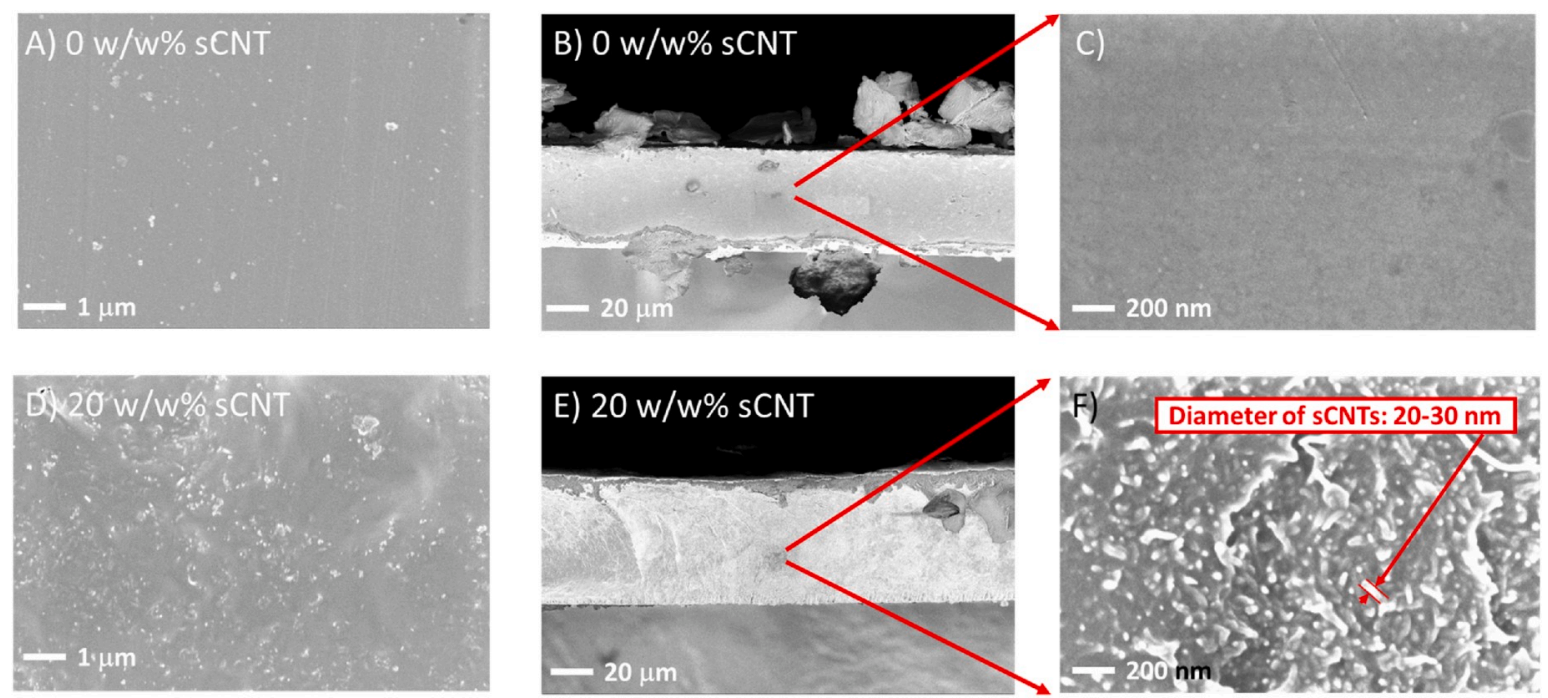

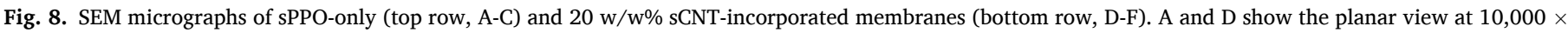

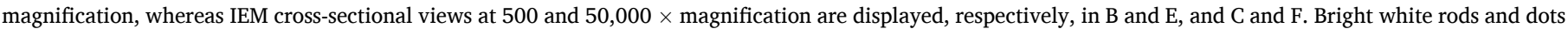
of 20-30 nm width in D-F are sCNTs (due to electrical conductivity).

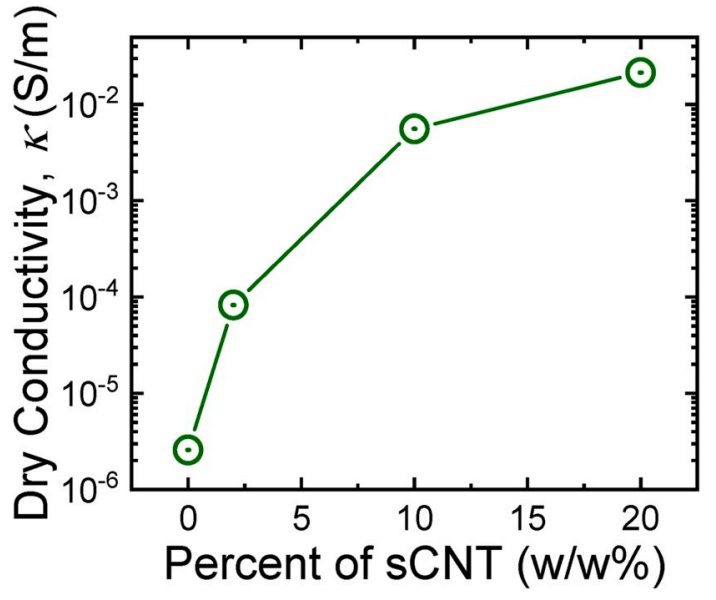

Fig. 9. Conductivity of dry membranes measured by electrochemical impedance spectroscopy, $\kappa$ (on a logarithmic scale), as a function of weight percent of sCNT incorporation. From the Nyquist plots (not shown), the impedances of 0 and $2.0 \mathrm{w} / \mathrm{w} \%$ sCNT membranes have contribution from both resistance and capacitive reactance, whereas the impedances of 10 and $20 \mathrm{w} / \mathrm{w} \%$ sCNT membranes are close to an ideal resistor.

characterization of dry state membranes and cross-sectional SEM micrograph analysis corroborate that a percolating nanotube network was achieved in 10 and $20 \mathrm{w} / \mathrm{w} \%$ sCNT IEMs.

Based on the structure-property analyses of SEM imaging and EIS conductivity characterization, we deduce that the improved conductivity-permselectivity performance at adequately high 1D nanomaterial loading is due to the formation of a percolated CNT network when the filler exceeds the percolation threshold. We postulate that the contiguous and interconnected network of nanotubes across the nanocomposite IEM thickness favorably shortens the effective transport pathway for ions (illustrated by Fig. 10). In pristine membranes, i.e., without SCNT incorporation, the polymer chains are entangled, thus forming compact clusters [64]. We conjecture that the sCNTs embedded in the polymer matrix disrupt the compact polymer packing, loosening the structure to create shortcuts for ion electro-diffusion. Thus, the transport pathway is less tortuous and the effective ion diffusivity is correspondingly improved. In addition, the sCNTs of $5-10 \mathrm{~nm}$ inner

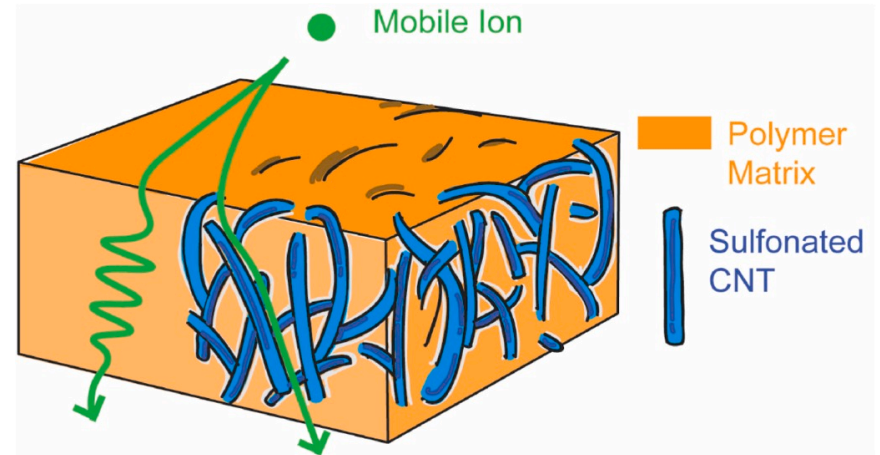

Fig. 10. Schematic illustrating ion transport in pristine and nanocomposite IEMs, with the polymer matrix represented in orange and sulfonated CNTs filler in the nanocomposite membrane denoted by blue tubes. The transport pathway of a mobile ion, depicted by the green sphere, across the nanotube-incorporated composite membrane is less tortuous compared to polymer-only matrix, as described by the green lines. (For interpretation of the references to colour in this figure legend, the reader is referred to the Web version of this article.)

diameter can function as fast ion conduction channels by allowing transport of hydrated ions inside the inner most tube [65], possibly contributing to the improvement of ion permeability.

Permselectivity, on the other hand, is not significantly impacted by the CNT incorporation. This is because the sulfonic acid moieties functionalized onto the sCNTs contribute to the IEC/fixed charge density of the membrane. In contrast, control membranes incorporated with $20 \mathrm{w} /$ w\% pristine CNTs, i.e., carbon nanotubes without negatively charged sulfonate moieties, possess appreciably lower permselectivity (approximately $-4 \%$ ) compared to IEMs with $20 \mathrm{w} / \mathrm{w} \%$ sCNT membranes under the same fabrication conditions. The lowered $\alpha$ of the pCNT nanocomposite IEMs can lead to an additional $20 \%$ co-ion leakage in electrodialysis-based applications, relative to membranes incorporated with sCNT. The charge exclusion effect of nanocomposite membranes with sulfonated carbon nanotubes is better preserved and, hence, selectivity for counterions is not detrimentally affected.

\section{Concluding remarks}

In this study, we developed novel nanocomposite ion exchanges 
membranes with percolated 1D nanomaterial network, i.e., sulfonated PPO polymer matrix incorporated with sulfonated carbon nanotube fillers. Unlike common conventional IEM fabrication approaches that tune the performance parameters through altering IEC or SD $[1,15]$, the nanocomposite membranes with percolated nanotubes fabricated here demonstrate a new strategy to reduce membrane resistance while maintaining permselectivity, to advance the overall conductivity-permselectivity tradeoff line. The intrinsic resistivity of the nanocomposite IEMs is favorably lowered by as much as $25 \%$ with up to $20 \mathrm{w} / \mathrm{w} \%$ incorporation of sCNT. The percolation of carbon nanotubes inside the nanocomposite membranes is corroborated by scanning electron microscope analysis and electrochemical impedance spectroscopy characterization. We postulate that the increase in effective ion conductivity is due to the interconnected network of sCNT reducing the tortuosity of the ion diffusion path. This study demonstrates the potential of rational utilization of nanomaterials to advance the conductivity-permselectivity tradeoff governing conventional IEMs. However, additional investigations are needed to demonstrate that the approach can be extended to improve current best-performing IEMs (indicated by green dotted line of Fig. 7). Future studies to investigate the effect of nanotubes properties, such as, diameter, length, functionality, and aspect ratio, can further enhance the performance of nanocomposite ion-exchange membranes. The IEM fabrication platform can be extended to other nanomaterials, such as nano-dots, -wires, and -sheets, to produce different matrix-filler nanocomposites.

\section{CRediT authorship contribution statement}

Hanqing Fan: Conceptualization, Methodology, Formal analysis, Investigation, Data curation, Software, Writing - original draft, Writing review \& editing, Visualization. Yuxuan Huang: Methodology, Data curation, Formal analysis, Investigation, Writing - original draft, Writing - review \& editing. Ngai Yin Yip: Conceptualization, Methodology, Formal analysis, Writing - original draft, Writing - review \& editing, Funding acquisition, Supervision.

\section{Acknowledgement}

We acknowledge financial support from the United States Bureau of Reclamation, Grant R16AC00124. We are grateful to Dr. Qian Cheng and Jonathan T. Vardner for the helpful discussions on EIS analysis, and to Kruti Sutaria for the assistance in manuscript preparation.

\section{References}

[1] H. Strathmann, Ion-exchange Membrane Separation Processes, Elsevier, 2004.

[2] H. Strathmann, Electrodialysis, a mature technology with a multitude of new applications, Desalination 264 (2010) 268-288.

[3] S. Bose, T. Kuila, T.X.H. Nguyen, N.H. Kim, K.-t. Lau, J.H. Lee, Polymer membranes for high temperature proton exchange membrane fuel cell: recent advances and challenges, Prog. Polym. Sci. 36 (2011) 813-843.

[4] G. Merle, M. Wessling, K. Nijmeijer, Anion exchange membranes for alkaline fuel cells: a review, J. Membr. Sci. 377 (2011) 1-35.

[5] P. Długołecki, K. Nymeijer, S. Metz, M. Wessling, Current status of ion exchange membranes for power generation from salinity gradients, J. Membr. Sci. 319 (2008) 214-222.

[6] T. Xu, Ion exchange membranes: state of their development and perspective, J. Membr. Sci. 263 (2005) 1-29.

[7] E. Güler, R. Elizen, D.A. Vermaas, M. Saakes, K. Nijmeijer, Performancedetermining membrane properties in reverse electrodialysis, J. Membr. Sci. 446 (2013) 266-276.

[8] N.Y. Yip, M. Elimelech, Comparison of energy efficiency and power density in pressure retarded osmosis and reverse electrodialysis, Environ. Sci. Technol. 48 (2014) 11002-11012.

[9] N.Y. Yip, D.A. Vermaas, K. Nijmeijer, M. Elimelech, Thermodynamic, energy efficiency, and power density analysis of reverse electrodialysis power generation with natural salinity gradients, Environ. Sci. Technol. 48 (2014) 4925-4936.

[10] P. Długołęcki, P. Ogonowski, S.J. Metz, M. Saakes, K. Nijmeijer, M. Wessling, On the resistances of membrane, diffusion boundary layer and double layer in ion exchange membrane transport, J. Membr. Sci. 349 (2010) 369-379.
[11] E. Guler, Y. Zhang, M. Saakes, K. Nijmeijer, Tailor-made anion-exchange membranes for salinity gradient power generation using reverse electrodialysis, ChemSusChem 5 (2012) 2262-2270.

[12] X. Tong, B. Zhang, Y. Chen, Fouling resistant nanocomposite cation exchange membrane with enhanced power generation for reverse electrodialysis, J. Membr. Sci. 516 (2016) 162-171.

[13] D.A. Vermaas, M. Saakes, K. Nijmeijer, Power generation using profiled membranes in reverse electrodialysis, J. Membr. Sci. 385 (2011) 234-242.

[14] H. Fan, N.Y. Yip, Elucidating conductivity-permselectivity tradeoffs in electrodialysis and reverse electrodialysis by structure-property analysis of ionexchange membranes, J. Membr. Sci. 573 (2019) 668-681.

[15] J. Ran, L. Wu, Y. He, Z. Yang, Y. Wang, C. Jiang, L. Ge, E. Bakangura, T. Xu, Ion exchange membranes: new developments and applications, J. Membr. Sci. 522 (2017) 267-291.

[16] S. Takamuku, A. Wohlfarth, A. Manhart, P. Räder, P. Jannasch, Hypersulfonated polyelectrolytes: preparation, stability and conductivity, Polym Chem-Uk 6 (2015) 1267-1274.

[17] R. Nagarale, G. Gohil, V.K. Shahi, R. Rangarajan, Preparation and electrochemical characterizations of cation-exchange membranes with different functional groups, Colloid. Surface. Physicochem. Eng. Aspect. 251 (2004) 133-140.

[18] L. Wu, T. Xu, Improving anion exchange membranes for DMAFCs by intercrosslinking CPPO/BPPO blends, J. Membr. Sci. 322 (2008) 286-292.

[19] S. Hosseini, S. Madaeni, A. Khodabakhshi, Preparation and characterization of ABS/HIPS heterogeneous cation exchange membranes with various blend ratios of polymer binder, J. Membr. Sci. 351 (2010) 178-188.

[20] Y. Xiong, J. Fang, Q.H. Zeng, Q.L. Liu, Preparation and characterization of crosslinked quaternized poly (vinyl alcohol) membranes for anion exchange membrane fuel cells, J. Membr. Sci. 311 (2008) 319-325.

[21] T. Sata, K. Teshima, T. Yamaguchi, Permselectivity between two anions in anion exchange membranes crosslinked with various diamines in electrodialysis, J. Polym. Sci. Polym. Chem. 34 (1996) 1475-1482.

[22] M.M. Pendergast, E.M. Hoek, A review of water treatment membrane nanotechnologies, Energy Environ. Sci. 4 (2011) 1946-1971.

[23] A. Alabi, A. AlHajaj, L. Cseri, G. Szekely, P. Budd, L. Zou, Review of nanomaterialsassisted ion exchange membranes for electromembrane desalination, npj Clean Water 1 (2018) 10.

[24] P.-C. Ma, N.A. Siddiqui, G. Marom, J.-K. Kim, Dispersion and functionalization of carbon nanotubes for polymer-based nanocomposites: a review, Compos. Appl. Sci. Manuf. 41 (2010) 1345-1367.

[25] W. Bauhofer, J.Z. Kovacs, A review and analysis of electrical percolation in carbon nanotube polymer composites, Compos. Sci. Technol. 69 (2009) 1486-1498.

[26] K.I. Winey, T. Kashiwagi, M. Mu, Improving electrical conductivity and thermal properties of polymers by the addition of carbon nanotubes as fillers, MRS Bull. 32 (2007) 348-353.

[27] K.I. Winey, R.A. Vaia, Polymer nanocomposites, MRS Bull. 32 (2007) 314-322.

[28] C. De Lannoy, D. Jassby, D. Davis, M. Wiesner, A highly electrically conductive polymer-multiwalled carbon nanotube nanocomposite membrane, J. Membr. Sci. 415 (2012) 718-724.

[29] G. He, Z. Li, J. Zhao, S. Wang, H. Wu, M.D. Guiver, Z. Jiang, Nanostructured ionexchange membranes for fuel cells: recent advances and perspectives, Adv. Mater. 27 (2015) 5280-5295.

[30] J.-M. Thomassin, J. Kollar, G. Caldarella, A. Germain, R. Jérôme, C. Detrembleur, Beneficial effect of carbon nanotubes on the performances of Nafion membranes in fuel cell applications, J. Membr. Sci. 303 (2007) 252-257.

[31] S. Yun, H. Im, Y. Heo, J. Kim, Crosslinked sulfonated poly (vinyl alcohol)/ sulfonated multi-walled carbon nanotubes nanocomposite membranes for direct methanol fuel cells, J. Membr. Sci. 380 (2011) 208-215.

[32] S. Hosseini, P. Koranian, A. Gholami, S. Madaeni, A. Moghadassi, P. Sakinejad, A. Khodabakhshi, Fabrication of mixed matrix heterogeneous ion exchange membrane by multiwalled carbon nanotubes: electrochemical characterization and transport properties of mono and bivalent cations, Desalination 329 (2013) 62-67.

[33] A. Zendehnam, S. Mokhtari, S. Hosseini, M. Rabieyan, Fabrication of novel heterogeneous cation exchange membrane by use of synthesized carbon nanotubes-co-copper nanolayer composite nanoparticles: characterization, performance in desalination, Desalination 347 (2014) 86-93.

[34] Y.-H. Su, Y.-L. Liu, Y.-M. Sun, J.-Y. Lai, D.-M. Wang, Y. Gao, B. Liu, M.D. Guiver, Proton exchange membranes modified with sulfonated silica nanoparticles for direct methanol fuel cells, J. Membr. Sci. 296 (2007) 21-28.

[35] P. Salarizadeh, M. Javanbakht, M. Abdollahi, L. Naji, Preparation, characterization and properties of proton exchange nanocomposite membranes based on poly (vinyl alcohol) and poly (sulfonic acid)-grafted silica nanoparticles, Int. J. Hydrogen Energy 38 (2013) 5473-5479.

[36] Y. Lu, Y. Liu, N. Li, Z. Hu, S. Chen, Sulfonated graphitic carbon nitride nanosheets as proton conductor for constructing long-range ionic channels proton exchange membrane, J. Membr. Sci. 601 (2020) 117908.

[37] H. Beydaghi, M. Javanbakht, A. Bagheri, P. Salarizadeh, H. Ghafarian-Zahmatkesh, S. Kashefi, E. Kowsari, Novel nanocomposite membranes based on blended sulfonated poly (ether ether ketone)/poly (vinyl alcohol) containing sulfonated graphene oxide/Fe 3 O 4 nanosheets for DMFC applications, RSC Adv. 5 (2015) $74054-74064$.

[38] H. Beydaghi, M. Javanbakht, Aligned nanocomposite membranes containing sulfonated graphene oxide with superior ionic conductivity for direct methanol fuel cell application, Ind. Eng. Chem. Res. 54 (2015) 7028-7037.

[39] T. Luo, Y. Zhang, H. Xu, Z. Zhang, F. Fu, S. Gao, A. Ouadah, Y. Dong, S. Wang, C. Zhu, Highly conductive proton exchange membranes from sulfonated 
polyphosphazene-graft-copolystyrenes doped with sulfonated single-walled carbon nanotubes, J. Membr. Sci. 514 (2016) 527-536.

[40] H. Bai, H. Zhang, Y. He, J. Liu, B. Zhang, J. Wang, Enhanced proton conduction of chitosan membrane enabled by halloysite nanotubes bearing sulfonate polyelectrolyte brushes, J. Membr. Sci. 454 (2014) 220-232.

[41] Y. Zhao, K. Tang, H. Ruan, L. Xue, B. Van der Bruggen, C. Gao, J. Shen, Sulfonated reduced graphene oxide modification layers to improve monovalent anions selectivity and controllable resistance of anion exchange membrane, J. Membr. Sci. 536 (2017) 167-175.

[42] Y. Wei, X. Ling, L. Zou, D. Lai, H. Lu, Y. Xu, A facile approach toward preparation of sulfonated multi-walled carbon nanotubes and their dispersibility in various solvents, Colloid. Surface. Physicochem. Eng. Aspect. 482 (2015) 507-513.

[43] T. Xu, W. Yang, Fundamental studies on a novel series of bipolar membranes prepared from poly (2, 6-dimethyl-1, 4-phenylene oxide)(PPO): I. Effect of anion exchange layers on I-V curves of bipolar membranes, J. Membr. Sci. 238 (2004) 123-129.

[44] C.G. Arges, L. Wang, J. Parrondo, V. Ramani, Best practices for investigating anion exchange membrane suitability for alkaline electrochemical devices: case study using quaternary ammonium poly (2, 6-dimethyl 1, 4-phenylene) oxide anion exchange membranes, J. Electrochem. Soc. 160 (2013) F1258.

[45] J.G. Hong, Y. Chen, Nanocomposite reverse electrodialysis (RED) ion-exchange membranes for salinity gradient power generation, J. Membr. Sci. 460 (2014) $139-147$.

[46] R. Huang, J. Kim, Synthesis and transport properties of thin film composite membranes. I. Synthesis of poly (phenylene oxide) polymer and its sulfonation, J. Appl. Polym. Sci. 29 (1984) 4017-4027.

[47] D. Wu, L. Wu, J.-J. Woo, S.-H. Yun, S.-J. Seo, T. Xu, S.-H. Moon, A simple heat treatment to prepare covalently crosslinked membranes from sulfonated poly $(2,6$ dimethyl-1, 4-phenylene oxide) for application in fuel cells, J. Membr. Sci. 348 (2010) 167-173.

[48] B. Gupta, F.N. Büchi, G.G. Scherer, A. Chapiró, Crosslinked ion exchange membranes by radiation grafting of styrene/divinylbenzene into FEP films, J. Membr. Sci. 118 (1996) 231-238.

[49] T. Sata, Ion Exchange Membranes: Preparation, Characterization, Modification and Application, Royal Society of chemistry, 2007.

[50] E. Barsoukov, J.R. Macdonald, Impedance Spectroscopy: Theory, Experiment, and Applications, John Wiley \& Sons, 2018.
[51] X. Qian, N. Gu, Z. Cheng, X. Yang, E. Wang, S. Dong, Impedance study of (PEO) 10LiClO4-Al2O3 composite polymer electrolyte with blocking electrodes, Electrochim. Acta 46 (2001) 1829-1836.

[52] R.S. Kingsbury, S. Flotron, S. Zhu, D.F. Call, O. Coronell, Junction potentials bias measurements of ion exchange membrane permselectivity, Environ. Sci. Technol. 52 (2018) 4929-4936.

[53] Y. Ji, G.M. Geise, The role of experimental factors in membrane permselectivity measurements, Ind. Eng. Chem. Res. 56 (2017) 7559-7566.

[54] H.J. Cassady, E.C. Cimino, M. Kumar, M.A. Hickner, Specific ion effects on the permselectivity of sulfonated poly (ether sulfone) cation exchange membranes, J. Membr. Sci. 508 (2016) 146-152.

[55] G.M. Geise, H.J. Cassady, D.R. Paul, B.E. Logan, M.A. Hickner, Specific ion effects on membrane potential and the permselectivity of ion exchange membranes, Phys. Chem. Chem. Phys. 16 (2014) 21673-21681.

[56] H. Strathmann, L. Giorno, E. Drioli, Introduction to Membrane Science and Technology, Wiley-VCH Weinheim, 2011.

[57] J. Moulder, W. Stickle, P. Sobol, K. Bomben, Handbook of X-Ray Photoelectron Spectroscopy, in: J. Chastain (Ed.), Perkin-Elmer Corp., Eden Prairie, MN, 1992.

[58] G.M. Geise, M.A. Hickner, B.E. Logan, Ionic resistance and permselectivity tradeoffs in anion exchange membranes, ACS Appl. Mater. Interfaces 5 (2013) 10294-10301.

[59] W. Cui, J. Kerres, G. Eigenberger, Development and characterization of ionexchange polymer blend membranes, Separ. Purif. Technol. 14 (1998) 145-154.

[60] J.G. Hong, B. Zhang, S. Glabman, N. Uzal, X. Dou, H. Zhang, X. Wei, Y. Chen, Potential ion exchange membranes and system performance in reverse electrodialysis for power generation: a review, J. Membr. Sci. 486 (2015) 71-88.

[61] J. Mackie, P. Meares, The diffusion of electrolytes in a cation-exchange resin membrane I. Theoretical, Proc. Roy. Soc. Lond. Math. Phys. Sci. 232 (1955) 498-509.

[62] J. Li, P.C. Ma, W.S. Chow, C.K. To, B.Z. Tang, J.K. Kim, Correlations between percolation threshold, dispersion state, and aspect ratio of carbon nanotubes, Adv. Funct. Mater. 17 (2007) 3207-3215.

[63] H.J. Li, W. Lu, J. Li, X. Bai, C. Gu, Multichannel ballistic transport in multiwall carbon nanotubes, Phys. Rev. Lett. 95 (2005), 086601.

[64] N. Berezina, N. Kononenko, O. Dyomina, N. Gnusin, Characterization of ionexchange membrane materials: properties vs structure, Adv. Colloid Interface Sci. 139 (2008) 3-28.

[65] B. Corry, Designing carbon nanotube membranes for efficient water desalination, J. Phys. Chem. B 112 (2008) 1427-1434. 